\title{
Margaric Acid
}

National Cancer Institute

\section{Source}

National Cancer Institute. Margaric Acid. NCI Thesaurus. Code C68389.

A saturated long-chain fatty acid with a 17-carbon backbone. Marg aric acid is found naturally as a minor component of the fat and milk fat of ruminants. 